\title{
Integrating Non-Invasive Neuroimaging and Computer Log Data to Improve Understanding of Cognitive Processes
}

\author{
Leah Friedman \\ Drexel University \\ Philadelphia, PA \\ lmf323@drexel.edu \\ Erin Walker \\ University of Pittsburgh \\ Pittsburgh, PA \\ eawalker@pitt.edu
}

\author{
Ruixue Liu \\ Worcester Polytechnic Institute \\ Worcester, MA \\ rliu2@wpi.edu \\ Erin T. Solovey \\ Worcester Polytechnic Institute \\ Worcester, MA \\ esolovey@wpi.edu
}

\begin{abstract}
As non-invasive neuroimaging techniques become less expensive and more portable, we have the capability to monitor brain activity during various computer activities. This provides an opportunity to integrate brain data with computer log data to develop models of cognitive processes. These models can be used to continually assess an individual's changing cognitive state and develop adaptive human-computer interfaces. As a step in this direction, we have conducted a study using functional near-infrared spectroscopy (fNIRS) during the Sustained Attention to Response Task (SART) paradigm, which has been used in prior work to elicit mind wandering and to explore response inhibition. The goal with this is to determine whether fNIRS data can be used as a predictor of errors on the task. This would have implications for detecting similar cognitive processes in more realistic tasks, such as using a personal learning environment. Additionally, this study aims to test individual differences by correlating objective behavioral data and subjective self reports with activity in the medial prefrontal cortex (mPFC), associated with the brain's default mode network (DMN). We observed significant differences in the mPFC between periods prior to task error and periods prior to a correct response. These differences were particularly apparent amongst those individuals who performed poorly on the SART task and those who reported drowsiness. In line with previous work, these findings indicate an opportunity to detect and correct attentional shifts in individuals who need it most.
\end{abstract}

\section{CCS CONCEPTS}

- Human-centered computing $\rightarrow$ Empirical studies in HCI;

\section{KEYWORDS}

functional near-infrared spectroscopy, fNIRS, SART

Permission to make digital or hard copies of all or part of this work for personal or classroom use is granted without fee provided that copies are not made or distributed for profit or commercial advantage and that copies bear this notice and the full citation on the first page. Copyrights for components of this work owned by others than ACM must be honored. Abstracting with credit is permitted. To copy otherwise, or republish, to post on servers or to redistribute to lists, requires prior specific permission and/or a fee. Request permissions from permissions@acm.org.

MCPMD'18, October 16, 2018, Boulder, CO, USA

(C) 2018 Association for Computing Machinery.

ACM ISBN 978-1-4503-6072-2/18/10 ..\$15.00

https://doi.org/10.1145/3279810.3279854
ACM Reference Format:

Leah Friedman, Ruixue Liu, Erin Walker, and Erin T. Solovey. 2018. Integrating Non-Invasive Neuroimaging and Computer Log Data to Improve Understanding of Cognitive Processes. In Workshop on Modeling Cognitive Processes from Multimodal Data (MCPMD'18 ), October 16, 2018, Boulder, CO, USA. ACM, New York, NY, USA, Article 4, 5 pages. https://doi.org/10.1145/ 3279810.3279854

\section{INTRODUCTION}

Functional near-infrared spectroscopy (fNIRS) has increasingly been used to assess cognitive state in real-time during interactive computing tasks $[1,8]$. fNIRS is an emerging tool that emits nearinfrared wavelengths at a person's forehead. These wavelengths are either absorbed or reflected back to the device depending on hemodynamic activity in the frontal cortex. The reflected light can then be used to detect highly active regions in the frontal lobe during a given task.

Most fNIRS research focuses on detecting and understanding patterns of activation in the anterior prefrontal cortex (aPFC), where the device is most effective. Because many studies using fMRI have found a connection between activation in the aPFC and cognitive states relevant to interactive computing, there is an opportunity for improving understanding of human-computer interaction processes through fNIRS neuroimaging. Devices are far less restrictive than fMRI, portable, and less costly [8], and thus they represent a unique opportunity to study cognition during realistic interactive computing tasks. Using fNIRS, we can take steps to bridge the conceptual gap between cognitive states identified in highly controlled cognitive neuroscience studies and the more complex, high-level cognitive states investigated in most HCI research [5].

We see promise in the combination of computer log analysis and non-invasive neuroimaging during computer usage since both methods provide unique moment-to-moment information about the user. The continuous nature of physiological measurements allows us to fill in gaps in the log data with insights from brain data. In particular, we can compare patterns in brain data occurring during interactive computing tasks with those collected during well-studied controlled cognitive tasks with similar timing characteristics. By relating these well-studied tasks that elicit particular cognitive states with interactive computing tasks with similar patterns, we can begin to understand more about the cognitive processes occurring in human-computer interaction. In addition, detailed computer log 


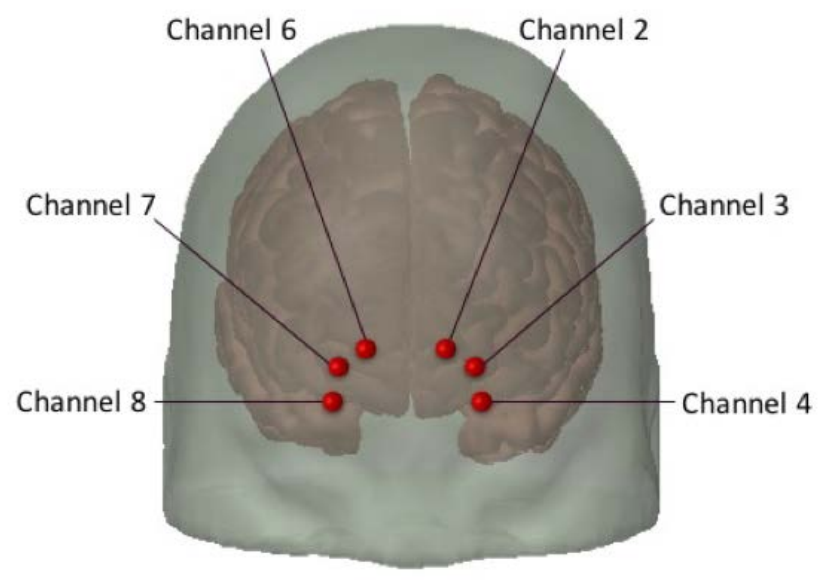

Figure 1: Approximate location of fNIRS channels in the anterior prefrontal cortex.

data provides contextual information that will enable better interpretations of the brain activity. Using brain-based sensing, our goal is to improve personalized interactive computing experiences.

To move towards these goals, we are building a corpus of combined brain and log data that enables us to model the user's cognitive state. We aim to collect data from both controlled lab tasks in which fNIRS is likely to detect distinct cognitive states as well as more realistic interactive computing tasks where those cognitive processes are also involved.

In this paper, we discuss initial work in which we explore the fNIRS response during a sustained attention to response task. Prior work [3] using fNIRS indicated significant differences in oxygenated hemoglobin $(\mathrm{HbO})$ in the mPFC between periods of successful and unsuccessful task completion. This was tied to mind wandering. In our study, we further investigated differences in frontal lobe blood oxygenation between successful and unsuccessful task completion states using fNIRS. We explore both behavioral data from the task and fNIRS data to gain insight into the cognitive processes occurring during this controlled task. This work serves as a foundation for modeling attentional states using machine learning tools to enable the creation of better and more personalized technology-based learning experiences.

\section{EXPERIMENT}

\subsection{Task}

A Sustained Attention to Response Task (SART) [6] was used while capturing frontal lobe activity with fNIRS. A number (1-9) was presented at the center of a blank white screen for 0.5 seconds followed by a 1.0 second pause. Participants were instructed to press the spacebar for each stimulus other than the number 3 (target stimulus). Stimuli were presented in 6 blocks, with 10 targets and 190 non-targets presented pseudorandomly in each block such that targets were not presented back to back.

\subsection{Equipment}

Hemodynamic data was collected with an 8-channel fNIRS device manufactured by ISS, Inc., with sensors placed against the participant's forehead and kept in place with headbands that reduced light interference. The SART program sent markers to an iMotions data acquisition platform, indicating the stimuli type (target or non-target). In addition, fNIRS raw data was sent in real-time via $\mathrm{TCP} / \mathrm{IP}$ to the iMotions platform, which synchronized the stimuli with the fNIRS signal.

\subsection{Participants}

There were 12 participants ( 8 female) between the ages of 18-41 (Mean: 28, SD: 6.48).

\subsection{Procedure}

After providing informed consent, each participant was given instructions about the SART task and the opportunity to ask questions. Then, the fNIRS sensors were positioned on the participant's head and they performed the SART task on a computer. The experiment consisted of six sections of 200 trials, with 10 targets in each trial section. Target trials were arranged pseudorandomly, such that no two target trials were immediately next to each other. In between sections, the subject was given a short ten second break.

At the end of the experiment, participants completed a postexperiment survey where they were asked to report their level of focus (scale of 1-7) and frequency of drowsiness and unrelated thoughts (scale from 'never' to 'very frequently', later converted to scale of 1-7). The questionnaire also provided a space to report opinions on SART and any difficulties experienced during the task.

\section{RESULTS}

\subsection{Subjective Responses}

Survey responses were analyzed for both mean and median. Mean focus level was reported to be $4.55(\operatorname{StdDev}=0.34$, Median $=5)$, mean number of unrelated thoughts was reported to be 4.46 (StdDev $=0.40$, Median $=4)$, and mean drowsiness level was $3.27(\mathrm{SE}=.56$, Median $=3$ ).

\subsection{Behavioral Data}

We were interested in examining the behavioral and brain activity differences between correct target responses (refrained from key press, not mind wandering) and incorrect target responses (key press, mind wandering). Mean correct response accuracy for target and non-target stimuli was calculated along with $95 \%$ confidence intervals.

Across all six blocks, mean accuracy in target stimuli responses was .7041 (SE $=.0372$, Median = .6554), with a 95\% CI from .6223.7860 (Figure 2). This was significantly lower than mean accuracy for non-target stimuli (Mean $=.9493, \mathrm{SE}=.0226$ ).

SART accuracy on target stimuli was calculated as a function of the three scaled survey questions, splitting participants into two groups based on the median survey response. In other words, we looked at accuracy on SART for those that reported above the median survey score in comparison to those that reported below 


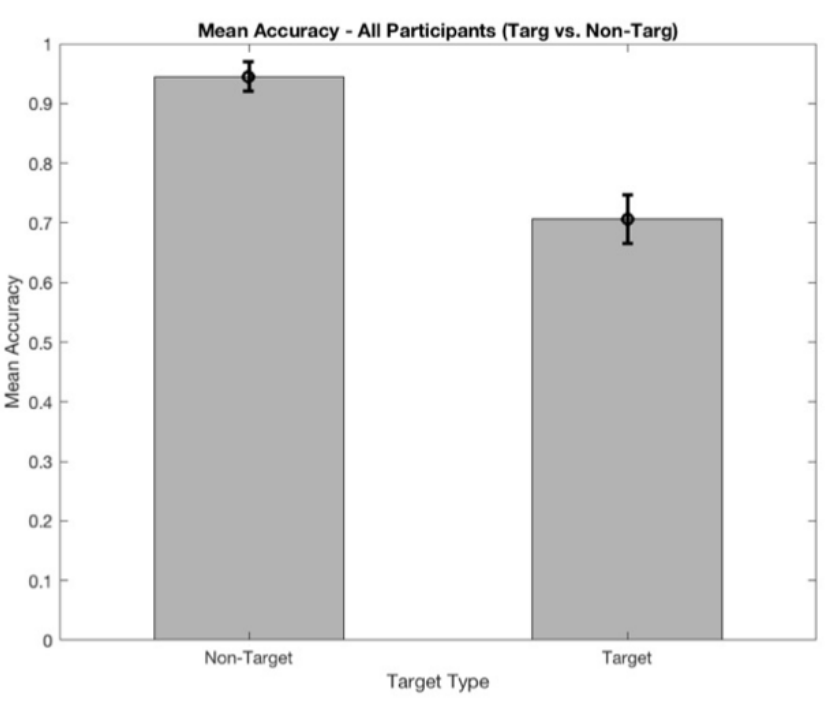

Figure 2: Average accuracy for SART Targets (Mean: .7041, SE: .0372) was significantly lower than average accuracy for Non-Targets (Mean: .9493, SE: .0226). Figure shows mean accuracy and standard error across all participants for SART Targets vs. SART non-targets.

the median. Participants that reported exactly the median survey score were included in the group above the median.

SART accuracy differed significantly for participants who reported above median drowsiness and those who reported below median drowsiness (Figure 5). SART accuracy on targets and nontarget did not significantly differ between participant who reported high and low focus levels and number of unrelated thoughts.

\section{3 fNIRS Data}

Raw fNIRS intensity values were converted to changes in oxygenated $(\mathrm{HbO})$ and deoxygenated $(\mathrm{HbR})$ hemoglobin using the modified Beer-Lambert Law [9]. All analysis was completed in MATLAB. For each target appearance, we extracted fNIRS data from 30 seconds prior to the target and 10 seconds after each target (referred to as target periods). It was possible that another target appeared in this 40-second window. Window length was informed by the work of Durantin et al. [3]. Target periods with significant artifacts were excluded using a threshold of $+/-2.0 \mu \mathrm{M}$. Data was downsampled to $2 \mathrm{~Hz}$ to account for slight variations in sample amount in the target periods. In order to assess change in $\mathrm{HbO}$ over time, we used the 5 seconds before each 40 -second window as a baseline. We calculated the average value in the baseline window and subtracted that value from the values in the 40-second window to center the data. Finally, we calculated the folded average of $\mathrm{HbO}$ change across all participants for the correct and incorrect target responses. Folded averages were also calculated along median splits for SART accuracy and survey responses. One participant was excluded from all analysis due to lack of in-range fNIRS data.

In fNIRS channels 2 and 3 (located around the medial to left prefrontal cortex), and 7 and 8 (located around the right anterior prefrontal cortex) (Figure 1), HbO decreased significantly in the 10
Mean HbO Change over all Participants during SART Error vs. No SART Error Periods
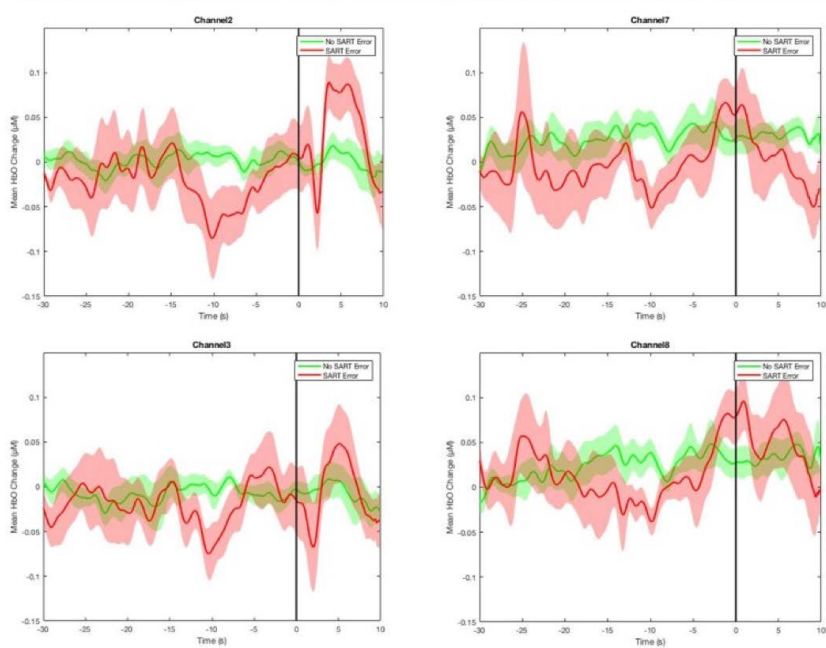

Figure 3: Average change in $\mathrm{HbO}$ showed a significant decrease in the 15 seconds preceding the appearance of a SART target in fNIRS channels 2,3,7, and 8. Figure shows averages across all target periods for all subjects for SART Errors (in red) and no SART Errors (in green). Shaded areas represent standard error for each trial type.

\section{Participants Split by Performance}
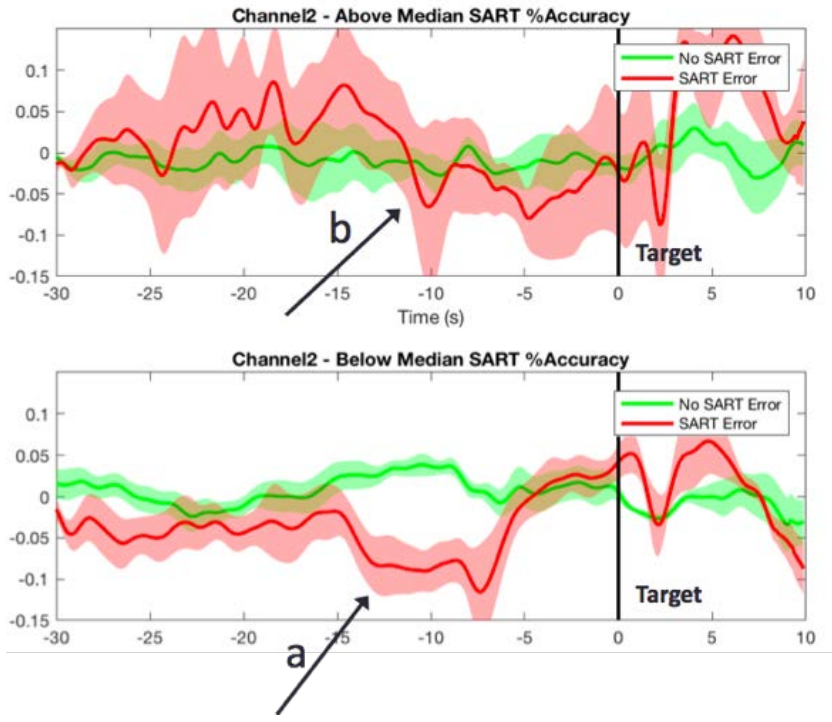

Figure 4: For participants with low task performance, the average change in $\mathrm{HbO}$ showed a significant decrease in the 15 seconds preceding the appearance of a SART target in fNIRS channels 2. Figure shows averages across all target periods for all subjects for SART Errors (in red) and no SART Errors (in green). Shaded areas represent standard error for each trial type. The bottom plot (a) shows only participants with below median task performance accuracy and the top figure (b) shows those with above median accuracy. 


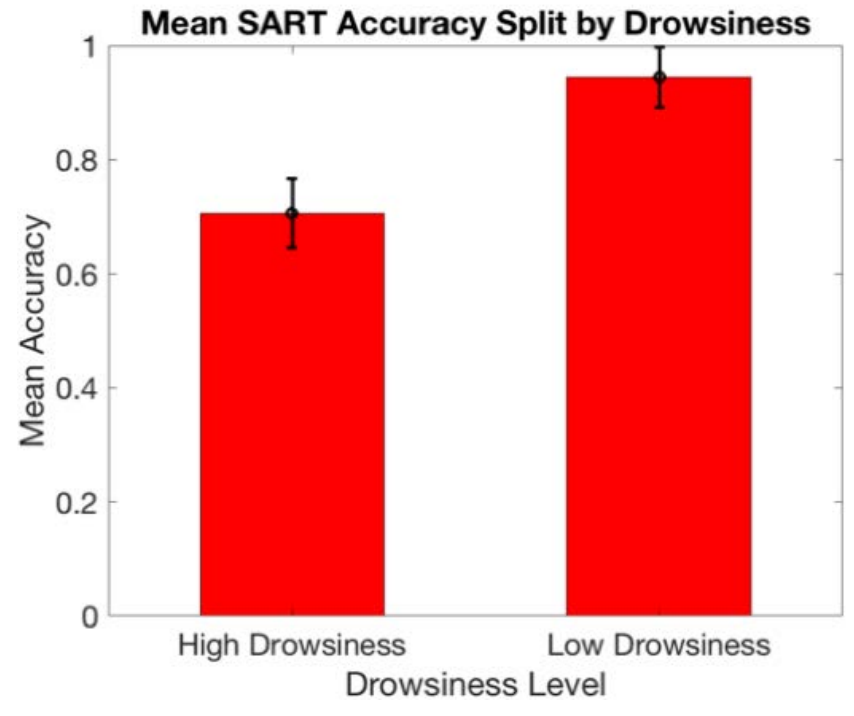

Figure 5: Mean accuracy on SART task, split by high and low drowsiness responses.

seconds preceding a missed target, and remained relatively constant in all channels during correct target hits (Figure 3). There were no significant differences in $\mathrm{Hb}$ over SART errors vs. no errors.

For participants that achieved below median accuracy on SART target responses (<.6554), fNIRS channels 2, 3, and 4 showed an even more significant $\mathrm{HbO}$ decrease in the 10 seconds preceding a missed target and again and remained relatively constant in all channels during correct target hits (Figure 4). For participants that achieved above median SART target accuracy (>.6554), there was no clear change or directionality in $\mathrm{HbO}$ in the target period.

Finally, in participants that reported above median drowsiness (>3), fNIRS channel 2 showed a similar dip in $\mathrm{HbO}$ in the 10 seconds preceding a missed target, while again remaining constant during target hits (Figure 6).

\section{DISCUSSION}

Consistent with prior work, we observed significantly lower response accuracy to target stimuli than non-target stimuli. Periods of poor performance on SART have been associated with mind wandering and have also been associated with activity in the default mode network, specifically in the medial prefrontal cortex as measured by stronger BOLD fMRI signal and increases in $\mathrm{HbO}$ $[2,3]$. Consistent with prior findings our work provides evidence that there are differences in frontal lobe blood oxygenation patterns between periods of mind wandering and periods of focus. Our findings are also consistent with those of Mason et al. [7], who found significantly stronger BOLD signal during mind wandering for individuals who self-reported higher mind wandering tendencies via the daydreaming frequency scale.

In contrast to previous findings that showed a significant increase in $\mathrm{HbO}$ immediately before the appearance of a target, our results showed a significant decrease in the 10 to 15 seconds preceding a target and a potential increase after the target. This result
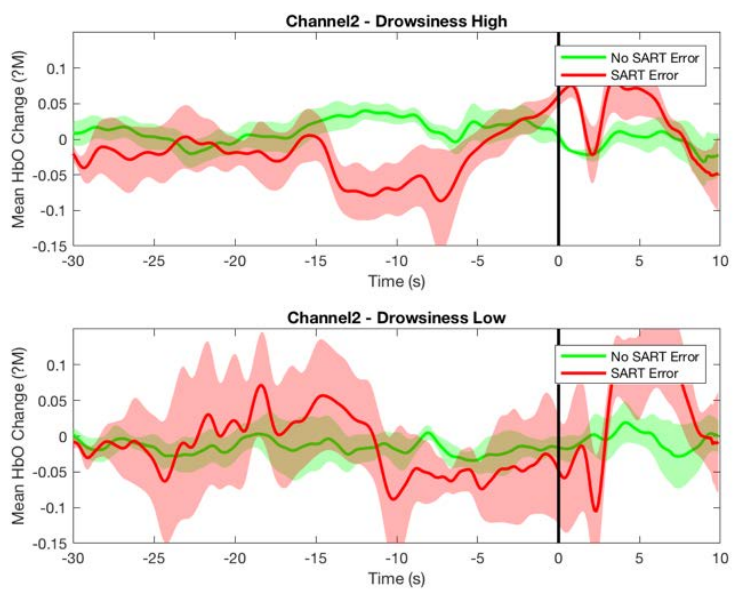

Figure 6: For participants that responded with high drowsiness levels, the average change in HbO showed a significant decrease in the 15 seconds preceding the appearance of a SART target in fNIRS channel 2 . Figure shows averages across all target periods for all subjects for SART Errors (in red) and no SART Errors (in green). Shaded areas represent standard error for each trial type.

might be accounted for by the hemodynamic response lag, meaning that event-related blood oxygenation may have occurred in the few seconds after the actual event. It is unclear whether prior work transformed data with a window shift and whether such a transformation would better align our data with the findings of Durantin et al. [3].

Furthermore, where Durantin et al. [3] noted temporal differences only in one optode near the medial prefrontal cortex located on the right of side of the prefrontal cortex, our preliminary results indicate that frontal lobe areas in both the left and right sides of the anterior prefrontal cortex may have distinct temporal patterns of $\mathrm{HbO}$ change during mind wandering, and should be considered when modeling mind wandering using machine learning. However, the medial prefrontal cortex was indeed the only area sensitive to poor performance on SART (as measured by channel 2 in our analysis). To build on these results, we also found that high levels of drowsiness also increases the visibility of the $\mathrm{HbO}$ decrease in the 10-15 seconds preceding a missed target.

\section{FUTURE WORK}

We plan to continue exploring this data through different signal processing methods, including noise reduction using short separation channels as has been described in recent work [4]. We also plan to correlate behavioral and fNIRS data to subjective responses from the post-experiment survey.

Using this behavioral and physiological dataset, we will develop machine learning approaches to robustly identify the cognitive states elicited by SART and other highly controlled tasks based on the multimodal data from fNIRS and behavioral data. The behavioral information gained can be used to generate labels associating a 
particular cognitive state (e.g. mind wandering) with fNIRS brain activity. With this labeled data from controlled tasks, we can explore supervised feature selection and classification models enabling the robust detection of the appropriate state.

\section{CONCLUSIONS}

These preliminary results demonstrate the possibility to detect hemodynamic changes that are predictive of task performance. The detection of predictors of performance without interruptions of the main task (e.g. experience sampling) builds a foundation for exploring brain patterns in more realistic and complex tasks during interactive computing tasks. These results show promise for the detection of adverse cognitive states in individuals that have the strongest tendency for these states.

\section{ACKNOWLEDGMENTS}

We would like to thank Aria Kim, Anush Lingamoorthy, Patricia Rahmlow, Gloria Houseman, Damian Baraty, Kyle Ellis, Juan Garcia Lopez, Denisa Qori and Reza Moradinezhad for their collaboration. This work was partially funded by Grants DGE-1835307 and CNS1711773 from the National Science Foundation as well as the CRA-W CREU project sponsored by the National Science Foundation.

\section{REFERENCES}

[1] Daniel Afergan, Evan M. Peck, Erin T. Solovey, Andrew Jenkins, Samuel W. Hincks, Eli T. Brown, Remco Chang, and Robert J.K. Jacob. 2014. Dynamic difficulty using brain metrics of workload. Proceedings of the 32nd annual ACM conference on Human factors in computing systems - CHI '14 (2014), 3797-3806. https://doi.org/ $10.1145 / 2556288.2557230$

[2] Kalina Christoff, Alan M Gordon, Jonathan Smallwood, Rachelle Smith, and Jonathan W Schooler. 2009. Experience sampling during fMRI reveals default network and executive system contributions to mind wandering. Proceedings of the National Academy of Sciences 106, 21 (2009), 8719-8724.

[3] Gautier Durantin, Frederic Dehais, and Arnaud Delorme. 2015. Characterization of mind wandering using fNIRS. Frontiers in systems neuroscience 9 (2015), 45.

[4] Louis Gagnon, Katherine Perdue, Douglas N Greve, Daniel Goldenholz, Gayatri Kaskhedikar, and David A Boas. 2011. Improved recovery of the hemodynamic response in diffuse optical imaging using short optode separations and state-space modeling. Neuroimage 56, 3 (2011), 1362-1371.

[5] Leanne M. Hirshfield, Erin Treacy Solovey, Audrey Girouard, James Kebinger Robert J.K. Jacob, Angelo Sassaroli, and Sergio Fantini. 2009. Brain measurement for usability testing and adaptive interfaces. In Proceedings of the 27th international conference on Human factors in computing systems - CHI 09. 2185. https://doi.org/ $10.1145 / 1518701.1519035$

[6] Tom Manly, Ian H Robertson, Maria Galloway, and Kari Hawkins. 1999. The absent mind:: further investigations of sustained attention to response. Neuropsychologia 37, 6 (1999), 661-670.

[7] Malia F Mason, Michael I Norton, John D Van Horn, Daniel M Wegner, Scott T Grafton, and C Neil Macrae. 2007. Wandering minds: the default network and stimulus-independent thought. science 315, 5810 (2007), 393-395.

[8] Erin Solovey, Paul Schermerhorn, Matthias Scheutz, Angelo Sassaroli, Sergio Fantini, and Robert Jacob. 2012. Brainput: Enhancing Interactive Systems with Streaming Fnirs Brain Input. Proceedings of the SIGCHI Conference on Human Factors in Computing Systems (2012), 2193-2202. https://doi.org/10.1145/2207676. 2208372

[9] Arno Villringer and Britton Chance. 1997. Non-invasive optical spectroscopy and imaging of human brain function. Trends in Neurosciences 20, 10 (1997), 435-442 https://doi.org/10.1016/S0166-2236(97)01132-6 